\title{
Keep ecological restoration open and flexible
}

To the Editor - The stakes are high as investment commitments to ecological restoration intensify ${ }^{1}$. The international standards' published by the Society for Ecological Restoration (SER $)^{2}$ and the adoption of a narrow definition of restoration by the United Nations Convention to Combat Desertification $(\mathrm{UNCCD})^{3}$ are indications that restoration is moving away from the open and flexible approach that we believe is needed to address responsible intervention in times of rapid change.

Restoration science originated in the idea that ecosystems could be returned to a defined pre-disturbance condition. This served as a proxy for improving biodiversity, structural attributes and functional traits, as well as for eliminating degradation. Now, three types of change are forcing restoration as a science and practice to reassess appropriate interventions in ecosystems. First, rapid environmental, ecological and cultural changes - ranging from climate change, to species invasions, to the rise of ecosystem-services valuation - are shifting the scope of traditional restoration. Second, innovation at the intersection of ecology, design and engineering is producing new types of ecosystem, such as green infrastructure, agroecosystems and naturalized landscapes. Third, investment in restoration has increased dramatically through major international agreements and conventions.

Restoration is positioned to become a go-to approach for addressing future environmental challenges by embracing a wide array of practices. This status must bring with it a signature commitment to ecosystem integrity, a disciplined approach to understanding the legacies and probable trajectories of ecosystems, and an appreciation of food security and human well-being. This may falter, however, if an overly narrow version of restoration is adopted.

There is evidence of this narrow approach emerging. For example, the Land Degradation Neutrality (LDN) framework of the UNCCD has adopted a relatively restrictive definition of restoration, emphasizing "pre-existing biotic integrity, in terms of species composition and community structure..."3. We argue that this characterization cedes too much to large-scale plantation forestry, revegetation, reclamation and rehabilitation approaches, which include little ambition for securing the ability of an ecosystem to support and maintain ecological processes and a diversity of organisms. Although these practices are useful in certain settings, they usually prioritize the realization of defined human expectations over ecological function and legacies. The restoration component of the LDN framework is based on SER's international standards ${ }^{2}$, which focus on 'substantial recovery' of 'appropriate local native reference ecosystems'. Under conditions of significant change, this stipulation will increasingly narrow the scope of restoration.

We urge professional organizations and governmental agencies, at all levels, to adopt restoration advice that embeds the capacity for practitioners and scientists to respond to ongoing and future change. Policy and operational guidance based on principles, guidelines and best practices suggest a path forward ${ }^{4}$. Broadly agreed principles would allow many more practices to be conducted within a restoration envelope, and still be anchored by strong ecological commitments and clear operational effectiveness ${ }^{5}$. We appreciate that the development of standards ${ }^{2}$ - comparative measures, norms or prescriptions - can be a useful element of restoration advice in ecosystems that are well-known or smaller in scale. But the fundamental changes described above contravene a one-size-fits-all approach. For standards to function well at any level there needs to be a much better-resolved scientific understanding of ecosystems, their natural dynamics and how they respond to change. As international and national organizations refine their policy advice, we encourage an open, flexible approach.

Eric S. Higgs ${ }^{1,2 \star}$, Jim A. Harris ${ }^{3}$, Tina Heger ${ }^{4}$, Richard J. Hobbs ${ }^{5}$, Stephen D. Murphy ${ }^{6}$ and Katharine N. Suding ${ }^{7}$

${ }^{1}$ School of Environmental Studies, University of Victoria, Victoria, BC V8P 5C2, Canada. ${ }^{2}$ Groningen Institute for Evolutionary Life Sciences, University of Groningen, Groningen, The Netherlands. ${ }^{3}$ Cranfield Soil and Agrifood Institute, Cranfield University, Cranfield MK43 OAL, UK.

${ }^{4}$ Institute for Biochemistry and Biology, University of Potsdam, Potsdam, Germany. ${ }^{5}$ School of Biological Sciences, University of Western Australia, Crawley, WA 6009, Australia. ${ }^{6}$ School of Environment, Resources and Sustainability, University of Waterloo, Waterloo, ON N2L 3G1, Canada. ${ }^{7}$ Department of Ecology and Evolutionary Biology, University of Colorado, Boulder, CO 80309, USA.

*e-mail: ehiggs@uvic.ca

Published online: 5 February 2018

https://doi.org/10.1038/s41559-018-0483-9

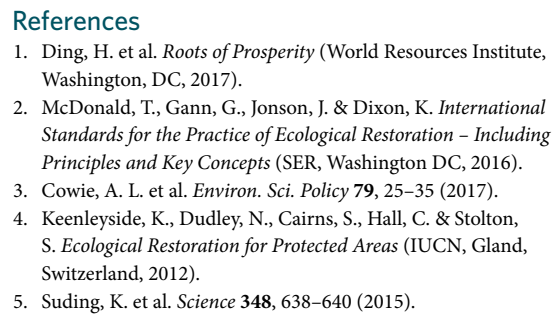

Competing interests

The authors declare no competing financial interests. 